\title{
Elevated serum fibroblast growth factor 21 levels correlate with immune recovery but not mitochondrial dysfunction in HIV infection
}

\author{
Brendan Al Payne ${ }^{1,2^{*}}$, David Ashley Price ${ }^{2}$ and Patrick F Chinnery ${ }^{1}$
}

\begin{abstract}
Background: Anti-retroviral treated HIV-infected patients are at risk of mitochondrial toxicity, but non-invasive markers are lacking. Serum FGF-21 (fibroblast growth factor 21) levels correlate strongly with muscle biopsy findings in inherited mitochondrial disorders. We therefore aimed to determine whether serum FGF-21 levels correlate with muscle mitochondrial dysfunction in HIV-infected patients.
\end{abstract}

Findings: We performed a cross-sectional study of anti-retroviral treated HIV-infected subjects (aged 29 - 71 years, $n=32$ ). Serum FGF-21 levels were determined by quantitative ELISA. Cellular mitochondrial dysfunction was assessed by COX (cytochrome c oxidase) histochemistry of lower limb skeletal muscle biopsy. Serum FGF-21 levels were elevated in $66 \%$ of subjects. Levels correlated significantly with current CD4 lymphocyte count $(p=0.042)$ and with total CD4 count gain since initiation of anti-retroviral therapy $(p=0.016)$, but not with the nature or duration of past or current anti-retroviral treatment. There was no correlation between serum FGF-21 levels and severity of the muscle mitochondrial (COX) defect.

Conclusions: Serum FGF-21 levels are a poor predictor of muscle mitochondrial dysfunction in contemporary anti-retroviral treated patients. Serum FGF-21 levels are nevertheless commonly elevated, in association with the degree of immune recovery, suggesting a non-mitochondrial metabolic disturbance with potential implications for future comorbidity.

Keywords: HIV, Anti-retroviral therapy, Highly active, Fibroblast growth factor 21, Mitochondria

\section{Findings}

Mitochondrial dysfunction is a well-described complication of anti-retroviral therapy [1-7]. It is most strongly associated with several of the older nucleoside analogue reverse transcriptase inhibitors (NRTIs): zidovudine (AZT), stavudine (d4T), didanosine (ddI), and zalcitabine $(\mathrm{ddC})$. Although these drugs are no longer in common usage in industrialised countries, there are nevertheless large numbers of patients who have had extensive prior exposure to these drugs, and some remain in common usage in developing countries. We have recently demonstrated that patients with previous exposure to these NRTIs may have

\footnotetext{
* Correspondence: brendan.Payne@ncl.ac.uk

'Institute of Genetic Medicine, Newcastle University, Central Parkway, Newcastle-upon-Tyne NE1 3BZ, UK

${ }^{2}$ Department of Infection and Tropical Medicine, Royal Victoria Infirmary, Newcastle-upon-Tyne NE1 4LP, UK
}

(c) 2013 Payne et al.; licensee BioMed Central Ltd. This is an open access article distributed under the terms of the Creative Commons Attribution License (http://creativecommons.org/licenses/by/2.0), which permits unrestricted use, distribution, and reproduction in any medium, provided the original work is properly cited.

persistent cellular mitochondrial COX (cytochrome $c$ oxidase) defects in skeletal muscle, consequent on an NRTI-induced accumulation of somatic (acquired) mitochondrial DNA (mtDNA) mutations [8].

Non-invasive measures of mitochondrial damage would be very valuable in the HIV clinic, both for the diagnosis of anti-retroviral associated mitochondrial dysfunction and the serial monitoring of such patients. The determination of mtDNA content in peripheral blood mononuclear cells (PBMCs) has previously been proposed as such a measure [5,9-11]. This consideration arises from the fact that the mitochondrially-toxic NRTIs (as listed above) cause a reduction in cellular mtDNA content (depletion) during therapy $[2,3,6,10,12-16]$. However, modern $\mathrm{N}(\mathrm{t})$ RTIs such as tenofovir (TDF) and abacavir (ABC) do not cause mtDNA depletion [17], and as a result, mtDNA levels return to normal with a switch away 
from a mitochondrially-toxic NRTI. Thus, measuring mtDNA levels is not a useful measure of on-going mitochondrial dysfunction due to an NRTI exposure in the distant past.

In contrast, FGF-21 (fibroblast growth factor 21) has recently been proposed as a valuable serum measure in inherited mitochondrial disease [18]. In these patients, serum FGF-21 levels showed a very strong correlation with mitochondrial dysfunction on skeletal muscle biopsy, as determined by the percentage of cells expressing a COX defect [18]. FGF-21 is thought to regulate mitochondrial activity and enhance oxidative capacity, mediated via PGC-1 $\alpha$ (peroxisome proliferator-activated receptor gamma co-activator 1-alpha) expression [19]. To date, one study has assessed serum FGF-21 in HIV infection, and demonstrated elevated levels [20]. Given the recently described association between serum FGF-21 elevation and muscle COX defects in inherited mitochondrial disorders [18], and the recent observation of significant COX defects in long-term anti-retroviral treated HIV-infected patients [8], we speculated that muscle mitochondrial dysfunction might also drive the FGF-21 elevation in anti-retroviral treated HIV infection.

\section{Patient characteristics}

All subjects gave informed written consent for participation, and the study was approved by local research ethics committee (Newcastle and North Tyneside Research Ethics Committee). We performed a crosssectional study of adult HIV-1 infected patients, receiving ambulatory care at one of two specialist clinics in Newcastle-upon-Tyne, UK. Patients with current active hepatitis $\mathrm{B}$ or $\mathrm{C}$ co-infection, known inherited or nonHIV-associated neuromuscular disease, and diabetes mellitus were excluded. No subjects were clinically obese (BMI >30). 32 HIV-infected subjects participated, of whom $81 \%$ were male. $84 \%$ were of white Caucasian ethnicity and the remainder black African. Mean age was 48.7 years, with age range of 29-71 years. Mean duration of diagnosed HIV infection was 10.8 years. Mean current CD4 lymphocyte count was 663 cells/ $\mu$ l, and $61 \%$ of subjects had nadir CD 4 count of $<200$ cells/ $\mu l$. All subjects were currently receiving combination anti-retroviral therapy, with a mean duration of treatment of 9.2 years. $97 \%$ of patients had fully suppressed HIV plasma viral load (<50 HIV-1 RNA copies/ml). 81\% of subjects were receiving a non-nucleoside reverse transcriptase inhibitor (NNRTI) and 22\% a ritonavirboosted protease inhibitor (PI). Regarding past (lifetime) NRTI treatment experience, $72 \%$ of patients had a history of AZT exposure, and $25 \%$ had a history of prior d-drug (dideoxynucleoside analogue) exposure. Characteristics of individual subjects are shown in Table 1.

\section{FGF-21 determination}

Serum FGF-21 levels were determined by quantitative ELISA (BioVendor, Brno, Czech Republic), performed in triplicate, and normalised by $\log _{10}$ transformation. A serum FGF-21 level of $<200 \mathrm{pg} / \mathrm{ml}$ was considered as normal in keeping with recent data [18]. Statistical analyses were performed in SPSS 19, using student's t-test to compare binary variables and Pearson's correlation coefficient ( $r$ ) to examine the relationship between $\log _{10}$ serum FGF-21 levels and continuous variables. Twentyone of 32 subjects (66\%) had serum FGF-21 levels greater than the normal range, with four being very elevated $(>800 \mathrm{pg} / \mathrm{ml})$. On univariate analysis, serum FGF-21 levels were positively correlated with current CD4 lymphocyte count $(\mathrm{r}=0.36, \mathrm{p}=0.042)$, but more strongly correlated with total CD4 cell count gain since initiation of anti-retroviral therapy (current minus nadir) $(\mathrm{r}=0.45, \mathrm{p}=0.016) \quad$ (Figure 1$)$. In addition, plasma glucose levels correlated with serum FGF-21 levels, although this did not quite reach statistical significance $(r=0.34, p=0.06$, Figure 2$)$, whereas as serum lipids and liver function did not. No other demographic or treatment variables were significantly associated with serum FGF-21 levels, including the nature of current or prior anti-retroviral therapy (Table 2). FGF-21 levels did not differ significantly between patients with or without clinical lipodystrophy syndrome. Only CD4 lymphocyte count gain was independently associated with serum FGF-21 levels on multivariate linear regression analysis $(\mathrm{p}=0.016)$.

\section{Skeletal muscle mitochondrial histochemistry}

COX histochemistry was performed on cryo-sections obtained from lower limb skeletal muscle biopsies on 31 of the 32 subjects (biopsy data for one subject was not analysable). Results of 22 of these biopsies have been reported in our previous work [8], whereas the remaining 9 have not. COX contains respiratory chain subunits encoded by the mitochondrial genome, and fibres stain brown (positive) in the presence of intact respiratory chain activity (Figure 3). Proportional COX defect was determined by counting $\geq 500$ fibres per biopsy, and normalised by $\log _{10}$ transformation. There was no correlation between serum FGF-21 levels and percentage COX defects on biopsy $(r=-0.02, p=0.9$, Figure 4).

\section{Discussion}

We have shown that serum FGF-21 levels are frequently elevated in contemporary anti-retroviral treated HIVinfected patients, but do not correlate with the severity of muscle mitochondrial (COX) defect. In contrast, a previous study has shown a very strong correlation between these parameters in patients with inherited mitochondrial disorders [18]. Ours is the first study to 
Table 1 Patient characteristics

\begin{tabular}{|c|c|c|c|c|c|c|c|c|c|c|c|}
\hline Age (y) & Gender & Ethnicity & $\begin{array}{c}\text { Duration of } \\
\text { diagnosed HIV (mo) }\end{array}$ & $\begin{array}{c}\text { ART } \\
\text { duration (mo) }\end{array}$ & $\begin{array}{c}\text { Nadir CD4 } \\
\text { count (cells/uL) }\end{array}$ & $\begin{array}{l}\text { Current CD4 } \\
\text { count (cells/uL) }\end{array}$ & LDS & ART (current) & ART (lifetime) & $\begin{array}{c}\text { COX } \\
\text { defect (\%) }\end{array}$ & $\begin{array}{l}\text { Serum FGF-21 } \\
(\mathrm{pg} / \mathrm{mL})\end{array}$ \\
\hline 71 & M & WB & 130 & 130 & UK & 530 & $\mathrm{Y}$ & TDF FTC EFV & ddi AZT 3TC EFV TDF FTC & $3.0 \%$ & 232 \\
\hline 48 & $\mathrm{~F}$ & BA & 100 & 99 & 10 & 487 & N & TDF FTC NVP & AZT 3TC EFVTDF FTC NVP & $0.1 \%$ & $>1920$ \\
\hline 34 & $\mathrm{~F}$ & WB & 88 & 86 & 218 & 1121 & Y & ABC 3TC NVP & AZT 3TC NVP ABC & $0.0 \%$ & 560 \\
\hline 55 & $\mathrm{~F}$ & BA & 64 & 27 & 112 & 426 & N & TDF FTC AZT DRV/r & TDF FTC LPV/r AZT DRV/r & $0.8 \%$ & 164 \\
\hline 43 & M & BA & 87 & 87 & 152 & 306 & N & TDF FTC EFV & AZT 3TC EFV TDF FTC & $0.8 \%$ & 20 \\
\hline 42 & M & WB & 185 & 147 & 150 & 636 & N & AZT 3TC EFV & AZT 3TC NVP & $0.0 \%$ & 342 \\
\hline 63 & M & WB & 97 & 97 & 169 & 870 & N & ABC 3TC EFV & AZT 3TC EFV ABC & $0.0 \%$ & 204 \\
\hline 29 & M & WB & 84 & 32 & 197 & 401 & N & TDF FTC EFV & TDF FTC EFV & $0.0 \%$ & 809 \\
\hline 63 & M & WB & 238 & 221 & NA & 438 & N & ABC $3 T C$ NVP & AZT ddl d4T 3TC ddC IDV NVP ABC & $2.2 \%$ & 440 \\
\hline 62 & M & WB & 63 & 62 & 56 & 190 & N & TDF FTC NVP & TOP FTC NVP & $0.2 \%$ & 156 \\
\hline 52 & M & WB & 225 & 225 & 120 & 728 & Y & TDF FTC ATV/r & $\begin{array}{l}\text { AZT ddC ddl 3TC d4T SQV NVP } \\
\text { IDV NFV ABC TDF LPV/r FTC ATV/r }\end{array}$ & $1.3 \%$ & 328 \\
\hline 36 & M & WB & 139 & 138 & 197 & 627 & N & AZT 3TC EFV & AZT 3TC EFV TDF FTC & $0.3 \%$ & 214 \\
\hline 51 & M & WB & 190 & 183 & 10 & 747 & N & ABC TDF NVP & $\begin{array}{l}\text { AZT ddl d4T 3TC RTV NVP } \\
\text { IDV ddC ABC ATV/r TDF }\end{array}$ & $4.9 \%$ & 252 \\
\hline 33 & $\mathrm{~F}$ & WB & 96 & 95 & 83 & 1289 & N & TDF FTC EFV & AZT 3TC EFV TDF FTC & $0.0 \%$ & 177 \\
\hline 48 & M & WB & 102 & 100 & 259 & 1329 & Y & AZT 3TC EFV & AZT 3TC EFV & $0.4 \%$ & 409 \\
\hline 51 & M & WB & 145 & 144 & 151 & 421 & N & AZT 3TC NVP & AZT 3TC NVP & $1.4 \%$ & 254 \\
\hline 66 & M & WB & 71 & 26 & 287 & 455 & N & TDF FTC EFV & TDF FTC EFV & $11.2 \%$ & 470 \\
\hline 46 & M & WB & 158 & 157 & 250 & 1452 & N & TDF FTC EFV & AZT 3TC IDV EFV ABC TDF FTC & $1.4 \%$ & 1218 \\
\hline 61 & M & WB & 116 & 113 & NA & 498 & Y & TDF FTC NVP & AZT 3TC EFV NVP TDF FTC & $2.4 \%$ & $182-$ \\
\hline 30 & M & WB & 88 & 23 & 283 & 661 & N & TDF FTC DRV/r & TDF FTC EFV DRV/r & $0.1 \%$ & 207 \\
\hline 62 & M & WB & 284 & 202 & NA & 422 & N & ABC NVP LPV/r & $\begin{array}{l}\text { SQV AZT ddC 3TC d4T ddl } \\
\text { IDV ABC NVP NFV LPV/r }\end{array}$ & $0.8 \%$ & 92 \\
\hline 45 & & WB & 159 & 158 & 176 & 660 & N & TDF FTC NVP & AZT 3TC IDV NVP TDF FTC & $0.0 \%$ & 60 \\
\hline 54 & M & WB & 79 & 38 & 244 & 638 & N & TDF FTC DRV/r & TDF FTC EFV DRV/r & $3.4 \%$ & 178 \\
\hline 52 & M & WB & 166 & 164 & 0 & 662 & Y & TDF FTC NVP & $\begin{array}{l}\text { AZT d4T IDV NFV SQV 3TC } \\
\text { NVP ddl TDF FTC }\end{array}$ & $2.8 \%$ & 525 \\
\hline 51 & M & WB & 243 & 171 & 327 & 539 & Y & TDF FTC EFV & AZT ddl RTV NFV TDF FTC EFV & $1.5 \%$ & 231 \\
\hline 35 & $\mathrm{~F}$ & BA & 62 & 25 & 380 & 638 & N & TDF FTC EFV & TOP FTC EFV & $0.0 \%$ & 82 \\
\hline 53 & M & WB & NA & 48 & 301 & 804 & N & TDF FTC EFV & TDF FTC EFV & NA & 490 \\
\hline 36 & M & WB & 130 & 130 & 18 & 898 & N & TDF FTC ATV/r & AZT 3TC EFV TDF FTC ATV/r & $0.0 \%$ & 530 \\
\hline 48 & M & WB & 53 & 14 & 332 & 443 & $\mathrm{~N}$ & TDF FTC EFV & TDF FTC EFV & $0.0 \%$ & 56 \\
\hline
\end{tabular}


Table 1 Patient characteristics (Continued)

\begin{tabular}{|c|c|c|c|c|c|c|c|c|c|c|c|}
\hline 52 & $\mathrm{~F}$ & $B A$ & 83 & 81 & 17 & 485 & $\bar{Y}$ & TDF FTC EFV & AZT 3TC EFV TDF FTC & $0.7 \%$ & 224 \\
\hline 38 & M & WB & 129 & 128 & 4 & 761 & Y & TDF FTC EFV & AZT 3TC EFV TDF FTC & $0.0 \%$ & 935 \\
\hline 47 & M & WB & 183 & 164 & 305 & 668 & Y & ABC RAL ATV/r & $\mathrm{d} 4 \mathrm{~T}$ 3TC NVP ddl IDV ABC ATV/r RAL & $9.8 \%$ & 114 \\
\hline
\end{tabular}

Summary characteristics of individual HIV-infected subjects. (WB, white British; BA, black African; ART, anti-retroviral therapy; LDS, lipodystrophy syndrome; AZT, zidovudine; d4T, stavudine; ddl, didanosine; ddC, zalcitabine; 3TC, lamivudine; ABC, abacavir; TDF, tenofovir; FTC, emtricitabine; EFV, efavirenz; NVP, nevirapine; ATV, atazanavir; DRV, darunavir; LPV, lopinavir; SQV, saquinavir; NFV, nelfinavir; IDV, indinavi; RTV, ritonavir at therapeutic dose; /r, ritonavir at pharmacokinetic boosting dose; RAL, raltegravir; COX, cytochrome c oxidase; FGF-21, fibroblast growth factor 21; NA, not available). 


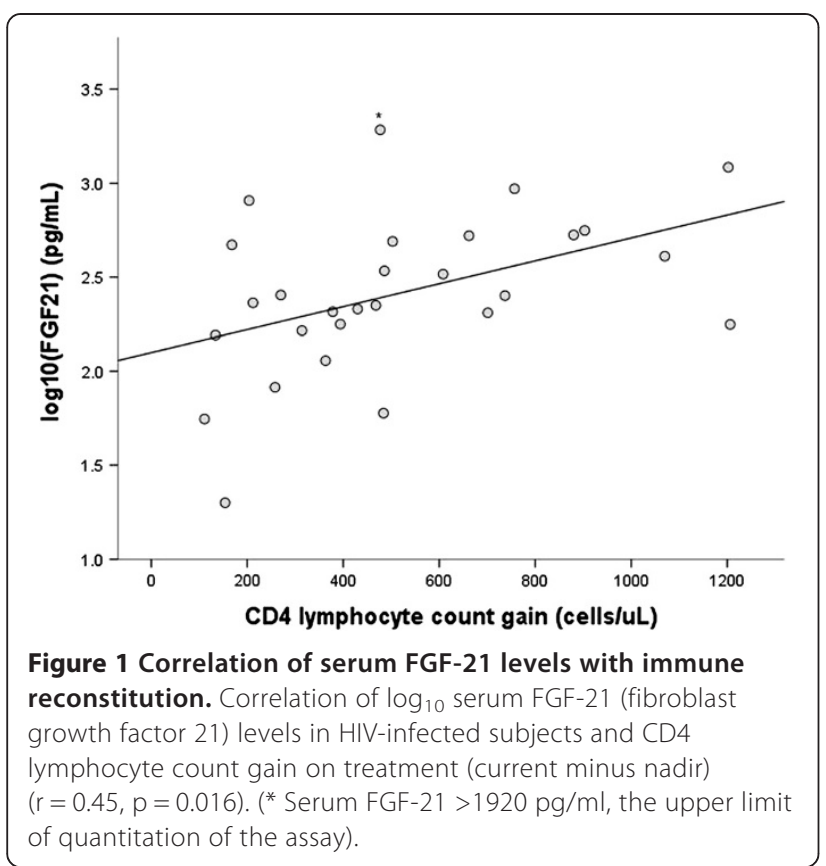

attempt to link serum FGF-21 levels with biopsyproven mitochondrial defects in HIV-infected patients. What is the reason for this apparent discrepancy in findings? Firstly, the prior study demonstrating serum FGF-21 elevation in mitochondrial disease included a large number of patients with childhood-onset disease. Such patients typically have very severe muscle COX defects (affecting up to $\sim 60 \%$ of fibres). In contrast,

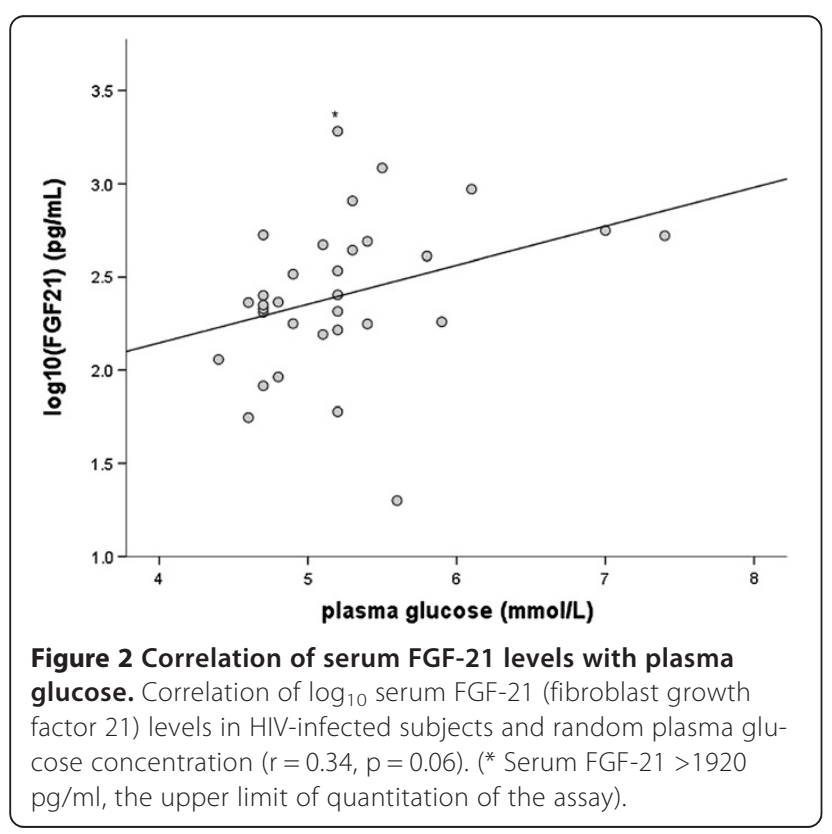

Table 2 Associations of serum FGF-21 levels

\begin{tabular}{|c|c|c|c|}
\hline (a) & & & \\
\hline Variable & (n) & $\begin{array}{c}\log _{10} \text { Serum FGF-21, } \\
\text { mean (SD) }\end{array}$ & $\begin{array}{c}p \\
\text { value }\end{array}$ \\
\hline \multirow[t]{2}{*}{ Gender } & Male (26) & $2.39(0.40)$ & \\
\hline & Female (6) & $2.46(0.48)$ & 0.71 \\
\hline \multirow[t]{2}{*}{ Ethnicity } & Caucasian (27) & $2.44(0.33)$ & \\
\hline & $\begin{array}{c}\text { Black African } \\
\text { (5) }\end{array}$ & $2.21(0.72)$ & 0.27 \\
\hline \multirow[t]{2}{*}{ Current ART } & $\mathrm{PI}(7)$ & $2.29(0.26)$ & \\
\hline & No PI (25) & $2.43(0.44)$ & 0.43 \\
\hline \multirow[t]{4}{*}{ Lifetime ART } & d-drugs (8) & $2.38(0.26)$ & \\
\hline & No d-drugs (24) & $2.41(0.45)$ & 0.86 \\
\hline & AZT (23) & $2.44(0.42)$ & \\
\hline & No AZT (9) & $2.30(0.39)$ & 0.41 \\
\hline \multirow[t]{2}{*}{ Lipodystrophy } & Yes (10) & $2.50(0.27)$ & \\
\hline & No (22) & $2.36(0.46)$ & 0.39 \\
\hline \multirow{2}{*}{$\begin{array}{l}\text { Lipid-lowering } \\
\text { therapy }\end{array}$} & Yes (8) & $2.32(0.52)$ & \\
\hline & No (24) & $2.43(0.38)$ & 0.53 \\
\hline \multicolumn{4}{|l|}{ (b) } \\
\hline Variable & & $\begin{array}{l}\text { Correlation } \\
\text { coefficient }(r)\end{array}$ & $\begin{array}{c}p \\
\text { value }\end{array}$ \\
\hline Age & & -0.07 & 0.69 \\
\hline $\begin{array}{c}\text { Duration of diagnosed } \\
\text { HIV infection }\end{array}$ & & -0.08 & 0.67 \\
\hline \multirow{3}{*}{$\begin{array}{l}\text { Duration of } \\
\text { lifetime ART }\end{array}$} & Total & 0.12 & 0.50 \\
\hline & d-drug & 0.01 & 0.97 \\
\hline & $\mathrm{AZT}$ & 0.13 & 0.47 \\
\hline \multirow{2}{*}{$\begin{array}{l}\text { CD4 lymphocyte } \\
\text { count }\end{array}$} & Nadir & -0.29 & 0.14 \\
\hline & Current & 0.36 & 0.042 \\
\hline CD4 count gain & $\begin{array}{l}\text { (Current minus } \\
\text { nadir) }\end{array}$ & 0.45 & 0.016 \\
\hline Serum ALT & & 0.27 & 0.14 \\
\hline Plasma glucose & & 0.34 & 0.06 \\
\hline \multirow[t]{3}{*}{ Serum lipids } & $\begin{array}{c}\text { Total } \\
\text { cholesterol }\end{array}$ & -0.03 & 0.87 \\
\hline & $\begin{array}{c}\mathrm{HDL} \\
\text { cholesterol }\end{array}$ & 0.02 & 0.90 \\
\hline & $\begin{array}{l}\text { Non-HDL } \\
\text { cholesterol }\end{array}$ & -0.06 & 0.75 \\
\hline $\begin{array}{l}\text { Mitochondrial } \\
\text { histochemistry }\end{array}$ & $\begin{array}{l}\text { COX defect } \\
\left(\log _{10}\right)\end{array}$ & -0.02 & 0.91 \\
\hline
\end{tabular}

(a) binary variables; (b) continuous variables. (PI, protease inhibitor; AZT, zidovudine; d-drug, dideoxynucleoside analogue; ART, anti-retroviral therapy; $A L T$, alanine transaminase; $\mathrm{HDL}$, high density lipoprotein). Bold type indicates statistically significant associations. 


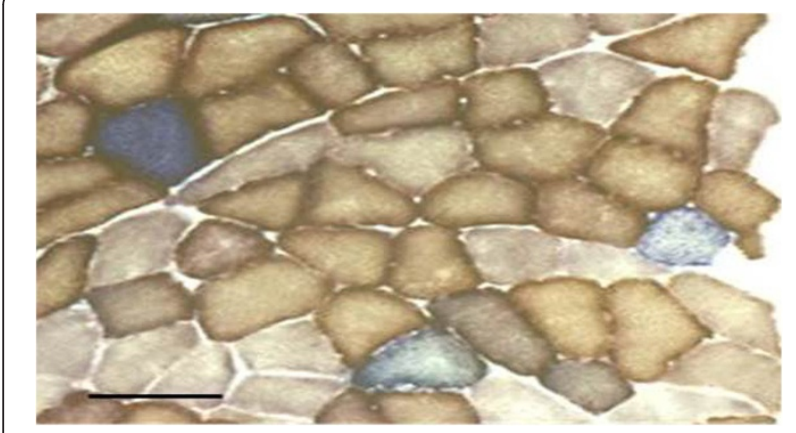

Figure 3 COX histochemistry. Example of mitochondrial COX/SDH (cytochrome c oxidase/succinate dehydrogenase) histochemistry on lower limb skeletal muscle biopsy of an anti-retroviral treated HIV-infected patient. Normal (COX positive) fibres stain brown, whereas COX deficient fibres counterstain blue due to preserved SDH activity.

patients with late-onset inherited mitochondrial disorders typically have more modest COX defects, comparable with those seen in our HIV-infected patients (up to $\sim 10 \%$ of fibres). The fact that some patients in our study with a biopsy COX defect of $>5 \%$ of fibres had relatively normal FGF-21 levels suggests that this serum measure is not particularly sensitive for mild to moderate muscle mitochondrial defects. Secondly, the markedly abnormal serum FGF-21 levels seen in some patients with no significant COX defect suggest a nonmitochondrial origin, as has been observed in other

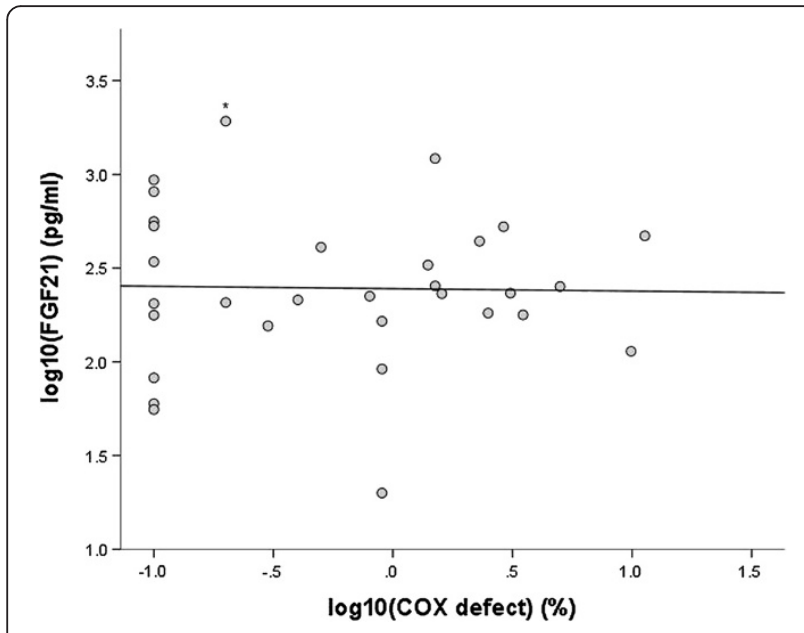

Figure 4 Correlation of serum FGF-21 levels and mitochondrial defects. Correlation of $\log _{10}$ serum FGF-21 (fibroblast growth factor 21) levels in HIV-infected subjects and percentage COX (cytochrome $c$ oxidase) defect on lower limb skeletal muscle biopsy $(r=-0.02$,

$p=0.9$ ). (* Serum FGF-21 $>1920 \mathrm{pg} / \mathrm{ml}$, the upper limit of quantitation of the assay). metabolic disorders [21-23]. In the only previous study of FGF-21 levels in HIV infection, the authors found associations of FGF-21 levels with obesity, glycaemia, dyslipidaemia and liver dysfunction, in line with literature from HIV-uninfected patients [24]. In our study, we specifically excluded diabetic and obese subjects (as we wished to maximise the likelihood of detecting any association with NRTI-induced mitochondrial dysfunction). Interestingly however, the strongest predictor of serum FGF-21 levels seen in our study was a novel association with total CD4 lymphocyte count gain. This is an intriguing finding. It is plausible that patients who have low nadir CD4 lymphocyte counts may experience more profound metabolic changes as they undergo immune reconstitution on anti-retroviral therapy, switching from a catabolic state to an excessively anabolic state associated with a 'return to health'. This association with CD4 count gain should be further explored by longitudinal study.

In conclusion, serum FGF-21 levels do not appear to be a sensitive or specific marker of muscle mitochondrial dysfunction in contemporary anti-retroviral treated patients. Nevertheless they are commonly elevated in association with immune recovery. As serum FGF-21 levels in the HIV-uninfected population are elevated in conditions associated with increased cardiovascular risk, it is very plausible that serum FGF-21 elevation in anti-retroviral treated HIV infection may also be a marker of an adverse metabolic risk in this patient group. Given the known increase in cardiovascular disease in anti-retroviral treated patients [25], the prognostic significance of our findings merits further research.

Competing interests

All authors confirm that they have no relevant competing interests.

\section{Authors' contributions}

BP conceived the study, performed the assays and drafted the manuscript. DAP conceived and helped coordinate the study. PFC conceived the study and drafted the manuscript. All authors read and approved the final manuscript.

\section{Acknowledgements}

We would like to acknowledge Alison Hague for assistance with the FGF-21 ELISA.

\section{Funding}

Medical Research Council (UK) (BP); Newcastle National Institute for Health Research (NIHR) Biomedical Research Centre in Ageing (BP, PFC); Medical Research Council (UK) Centre for Translational Muscle Disease (PFC); Wellcome Trust (084980/Z/08/Z \& 096919Z/11/Z, PFC). 


\section{References}

1. Dalakas MC, Illa I, Pezeshkpour GH, Laukaitis JP, Cohen B, Griffin JL: Mitochondrial myopathy caused by long-term zidovudine therapy. N Engl J Med 1990, 322:1098-1105.

2. Arnaudo E, Dalakas M, Shanske S, Moraes CT, DiMauro S, Schon EA: Depletion of muscle mitochondrial DNA in AIDS patients with zidovudine-induced myopathy. Lancet 1991, 337:508-510

3. Shikuma CM, Hu N, Milne C, Yost F, Waslien C, Shimizu S, Shiramizu B: Mitochondrial DNA decrease in subcutaneous adipose tissue of HIV-infected individuals with peripheral lipoatrophy. Aids 2001, 15:1801-1809

4. Zaera MG, Miro O, Pedrol E, Soler A, Picon M, Cardellach F, Casademont J, Nunes $\mathrm{V}$ : Mitochondrial involvement in antiretroviral therapy-related lipodystrophy. Aids 2001, 15:1643-1651.

5. van der Valk M, Casula M, Weverlingz GJ, van Kuijk K, van Eck-Smit B, Hulsebosch HJ, Nieuwkerk P, van Eeden A, Brinkman K, Lange J, et al: Prevalence of lipoatrophy and mitochondrial DNA content of blood and subcutaneous fat in HIV-1infected patients randomly allocated to zidovudine- or stavudine-based therapy. Antivir Ther 2004, 9:385-393.

6. Walker UA, Bauerle J, Laguno M, Murillas J, Mauss S, Schmutz G, Setzer B, Miquel R, Gatell JM, Mallolas J: Depletion of mitochondrial DNA in liver under antiretroviral therapy with didanosine, stavudine, or zalcitabine. Hepatology 2004, 39:311-317.

7. Miro O, Lopez S, Pedrol E, Rodriguez-Santiago B, Martinez E, Soler A, Milinkovic A, Casademont J, Nunes V, Gatell JM, Cardellach F: Mitochondrial DNA depletion and respiratory chain enzyme deficiencies are present in peripheral blood mononuclear cells of HIV-infected patients with HAART-related lipodystrophy. Antivir Ther 2003, 8:333-338.

8. Payne BA, Wilson IJ, Hateley CA, Horvath R, Santibanez-Koref M, Samuels DC, Price DA, Chinnery PF: Mitochondrial aging is accelerated by anti-retroviral therapy through the clonal expansion of mtDNA mutations. Nat Genet 2011, 43:806-810

9. Chiappini F, Teicher E, Saffroy R, Pham P, Falissard B, Barrier A, Chevalier S, Debuire B, Vittecoq D, Lemoine A: Prospective evaluation of blood concentration of mitochondrial DNA as a marker of toxicity in 157 consecutively recruited untreated or HAART-treated HIV-positive patients. Lab Invest 2004, 84:908-914.

10. Cote HC, Brumme ZL, Craib K, Alexander CS, Wynhoven B, Ting L, Wong H, Harris M, Harrigan PR, O'Shaughnessy MV, Montaner JS: Changes in mitochondrial DNA as a marker of nucleoside toxicity in HIV-infected patients. N Engl J Med 2002, 346:811-820.

11. Montaner JS, Cote HC, Harris M, Hogg RS, Yip B, Chan JW, Harrigan PR, O'Shaughnessy MV: Mitochondrial toxicity in the era of HAART: evaluating venous lactate and peripheral blood mitochondrial DNA in HIV-infected patients taking antiretroviral therapy. J Acquir Immune Defic Syndr 2003, 34(Suppl 1):S85-S90.

12. Cherry CL, Gahan ME, McArthur JC, Lewin SR, Hoy JF, Wesselingh SL: Exposure to dideoxynucleosides is reflected in lowered mitochondrial DNA in subcutaneous fat. J Acquir Immune Defic Syndr 2002, 30:271-277.

13. Hoschele D: Cell culture models for the investigation of NRTI-induced mitochondrial toxicity. Relevance for the prediction of clinical toxicity. Toxicol In Vitro 2006, 20:535-546.

14. Dalakas MC, Semino-Mora C, Leon-Monzon M: Mitochondrial alterations with mitochondrial DNA depletion in the nerves of AIDS patients with peripheral neuropathy induced by $2^{\prime} 3$ '-dideoxycytidine (ddC). Lab Invest 2001, 81:1537-1544.

15. Hammond E, Nolan D, James I, Metcalf C, Mallal S: Reduction of mitochondrial DNA content and respiratory chain activity occurs in adipocytes within 6-12 months of commencing nucleoside reverse transcriptase inhibitor therapy. Aids 2004, 18:815-817.

16. Lim SE, Copeland WC: Differential incorporation and removal of antiviral deoxynucleotides by human DNA polymerase gamma. J Biol Chem 2001, 276:23616-23623.

17. Birkus G, Hitchcock MJ, Cihlar T: Assessment of mitochondrial toxicity in human cells treated with tenofovir: comparison with other nucleoside reverse transcriptase inhibitors. Antimicrob Agents Chemother 2002, 46:716-723.

18. Suomalainen A, Elo JM, Pietilainen $\mathrm{KH}$, Hakonen AH, Sevastianova K, Korpela M, Isohanni P, Marjavaara SK, Tyni T, Kiuru-Enari S, et al: FGF-21 as a biomarker for muscle-manifesting mitochondrial respiratory chain deficiencies: a diagnostic study. Lancet Neurol 2011, 10:806-818.
19. Moyers JS, Shiyanova TL, Mehrbod F, Dunbar JD, Noblitt TW, Otto KA, Reifel-Miller A, Kharitonenkov A: Molecular determinants of FGF-21 activity-synergy and cross-talk with PPARgamma signaling. I Cell Physiol 2007, 210:1-6.

20. Affandi JS, Price P, Imran D, Yunihastuti E, Djauzi S, Cherry CL: Can we predict neuropathy risk before stavudine prescription in a resource-limited setting? AIDS Res Hum Retroviruses 2008, 24:1281-1284.

21. Chen WW, Li L, Yang GY, Li K, Qi XY, Zhu W, Tang Y, Liu H, Boden G: Circulating FGF-21 levels in normal subjects and in newly diagnose patients with Type 2 diabetes mellitus. Exp Clin Endocrinol Diabetes 2008, 116:65-68.

22. Dushay J, Chui PC, Gopalakrishnan GS, Varela-Rey M, Crawley M, Fisher FM, Badman MK, Martinez-Chantar ML, Maratos-Flier E: Increased fibroblast growth factor 21 in obesity and nonalcoholic fatty liver disease. Gastroenterology 2010, 139:456-463.

23. Zhang X, Yeung DC, Karpisek M, Stejskal D, Zhou ZG, Liu F, Wong RL, Chow WS, Tso AW, Lam KS, XU A: Serum FGF21 levels are increased in obesity and are independently associated with the metabolic syndrome in humans. Diabetes 2008, 57:1246-1253.

24. Domingo P, Gallego-Escuredo JM, Domingo JC, Gutierrez Mdel M, Mateo MG Fernandez I, Vidal F, Giralt M, Villarroya F: Serum FGF21 levels are elevated in association with lipodystrophy, insulin resistance and biomarkers of liver injury in HIV-1-infected patients. AIDS 2010, 24:2629-2637.

25. Friis-Moller N, Sabin CA, Weber R, D'Arminio Monforte A, El-Sadr WM, Reiss P, Thiebaut R, Morfeldt L, De Wit S, Pradier C, et al: Combination antiretroviral therapy and the risk of myocardial infarction. N Engl J Med 2003, 349:1993-2003.

doi:10.1186/1742-6405-10-27

Cite this article as: Payne et al: Elevated serum fibroblast growth factor 21 levels correlate with immune recovery but not mitochondrial dysfunction in HIV infection. AIDS Research and Therapy 2013 10:27.

\section{Submit your next manuscript to BioMed Central and take full advantage of:}

- Convenient online submission

- Thorough peer review

- No space constraints or color figure charges

- Immediate publication on acceptance

- Inclusion in PubMed, CAS, Scopus and Google Scholar

- Research which is freely available for redistribution
Ciomed Central 